\title{
Ultra-sharp soliton switching in a directional coupler
}

\author{
Theo P. Valkering, Joost van Honschoten, Hugo J.W.M. Hoekstra \\ Faculty of Applied Physics, University of Twente, P.O. Box 217, 7500 AE Enschede, The Netherlands
}

Received 1 July 1998; revised 5 October 1998; accepted 5 October 1998

\begin{abstract}
By a numerical investigation it is shown that a directional coupler, described by two linearly coupled non-linear Schrödinger equations, can be used to construct a soliton switch with an extremely narrow transition region. (C) 1999 Elsevier Science B.V. All rights reserved.
\end{abstract}

PACS: 42.65.Pc; 42.65.Tg

Keywords: Directional coupler; Soliton; All-optical switching; Coupled nonlinear Schrödinger equations

\section{Introduction}

In this paper we investigate in detail the switching process in a directional coupler via the coupled nonlinear Schrödinger equations. In Ref. [1] the geometrical background of this process was discussed for soliton switching. The numerical calculations in that paper had zero initial conditions in one channel and a soliton of given energy $N$ in the other. One of the results was that, with $N$ as the control parameter, the actual switching of the pulse takes place within an extremely small range of energy. In this paper this process is investigated in detail with the aim to see if a sharp routing device is feasible.

Switching was investigated by several authors, usually for so-called half beat length couplers. The emphasis was on the sharpness of the switching process. For surveys we refer to Refs. [2,3]. First experiments were reported in Ref. [4]. The effect of extra terms, such as gain or loss, in the standard coupler equations were considered in Ref. [5].

Detailed numerical and analytical investigations, including the use and meaning of low-dimensional models, were reported e.g. in Refs. [6,7]. See also Ref. [8] for a recent survey and more references relevant to the present subject.

For switching in the setting considered here a crucial property is that for the value of the coupling constant and for the range of energies of the input soliton considered, the symmetric soliton state (cf. Refs. $[9,8,10,11])$ is unstable. It can be shown [1] that the soliton has a one-dimensional unstable manifold, which seems to be the origin of the sharp switching reported in this paper.

\section{The equations}

The system of two coupled optical fibers will be modeled with the Coupled Non-Linear Schrödinger equations (CNLS), cf. Ref. [3]:

$$
\begin{aligned}
& u_{1 z}=i\left[\frac{1}{2} u_{1 t t}+\left|u_{1}\right|^{2} u_{1}+\kappa u_{2}\right], \\
& u_{2 z}=i\left[\frac{1}{2} u_{2 t t}+\left|u_{2}\right|^{2} u_{2}+\kappa u_{1}\right] .
\end{aligned}
$$

Here $\kappa$ is the coupling factor. The functions $u_{1}$ and $u_{2}$ are complex and defined on $-\infty<t<\infty$. They represent the envelopes of the electrical field in channels 1 and 2 of the coupler respectively. These functions as well as their first derivatives are supposed to be square integrable. The energies $N_{1,2}$ in each channel and the total energy are defined as

$N_{j}=\int_{-\infty}^{\infty} u_{j}^{*} u_{j} \mathrm{~d} t, \quad N=N_{1}+N_{2}$.

The analysis of CNLS can be simplified by using its similarity property: if $\underline{u}(t, z ; \kappa)$ is a solution for a gi- 
ven $\kappa$, then the scaled function $v(t, z ; \kappa, \alpha)=$ $\alpha u\left( \pm \alpha t, \alpha^{2} z ; \alpha^{-2} \kappa\right)$, where $\alpha$ is a real number, is a solution as well (for the same $\kappa$ ). Thus one solution generates a family of solutions depending on $\alpha$. One finds readily $N(v)=\alpha N(u)$, so that, if one fixes $N(u)=1, \alpha$ can be identified with $N$. Thus for representing our results it is useful to introduce the normalized quantities

$\hat{\kappa}=\frac{\kappa}{N^{2}}, \quad \hat{N}_{j}=\frac{N_{j}}{N}, \quad \hat{z}=N^{2} z$.

\section{Evolution of the relative energy $N(z)$ for increasing $\hat{\kappa}$}

Calculations were performed for initial conditions

$u_{1}=\frac{N}{2} \operatorname{sech}\left(\frac{N}{2} t\right), \quad u_{2}=0$.
The field in channel 1 is that of a soliton in a single fiber. In our calculations the field (4) is given at $z=0$, and the evolution of the signal is calculated along the coupled fibers, i.e. for $z>0 . N$ in (4) was given a fixed value, equal to 2 , and $\kappa$ was varied.

In Fig. 1 a series of $N$-evolutions are presented showing several aspects relevant for the use of the coupler as a switching device.

$(a),(b),(c),(d)$ : non-switching. In this quartet the signal energy does not switch in the range of $z$ shown. Clearly a smallest distance $\hat{z}_{\max }$ can be observed at which the relative energy $N_{1} / N$ attains a maximum, which corresponds to a minimum energy transfer between the channels. This distance $\hat{z}_{\max }$ is well defined, as only one maximum can be found in the present range of $z$. A second observation is that the relative energy $N_{1} / N$ reaches a value of about 0.9 . Finally, the maximum in these graphs (a)

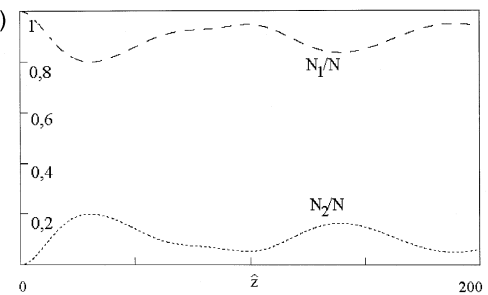

(d)

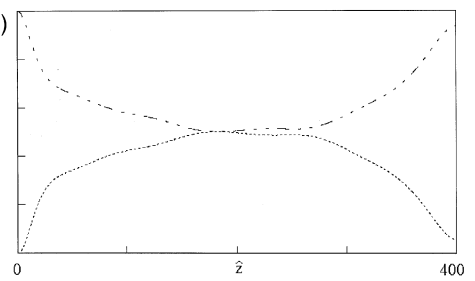

(g)

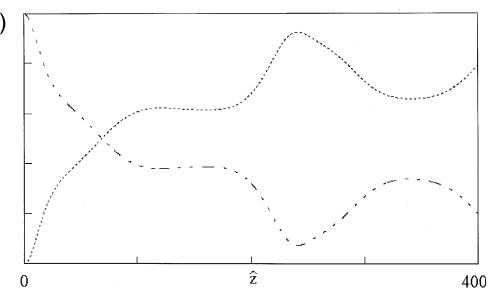

(j)

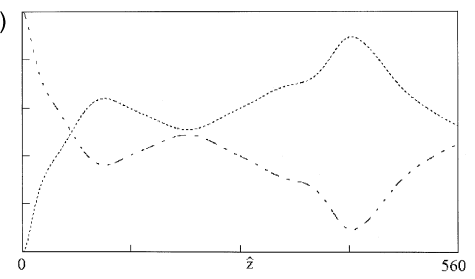

(b)

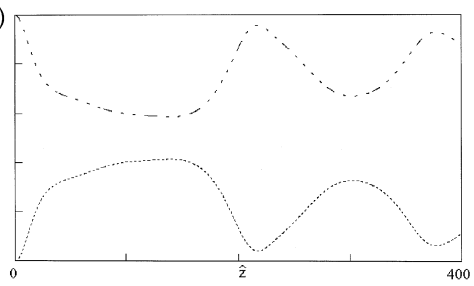

(e)

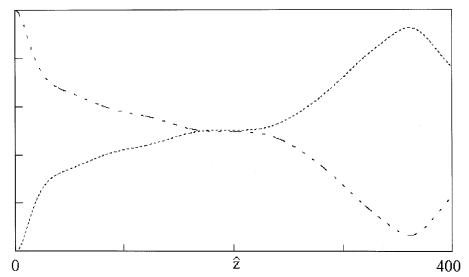

(h)
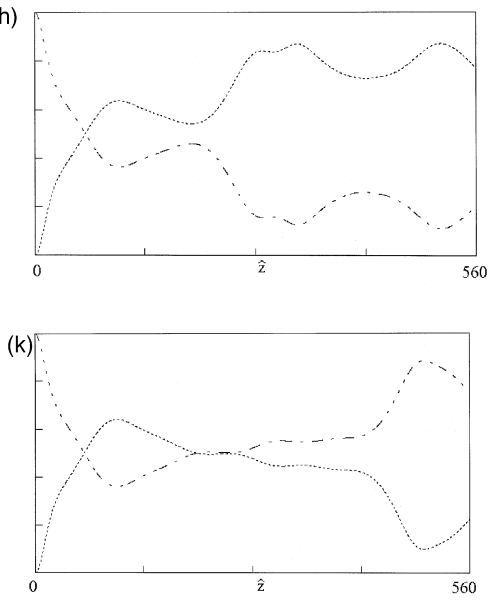

(c)

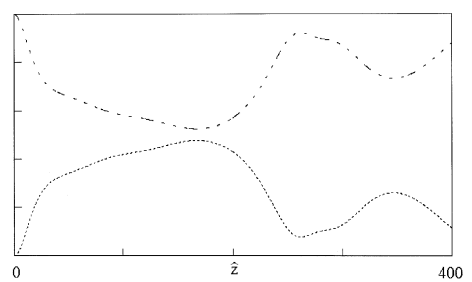

(f)

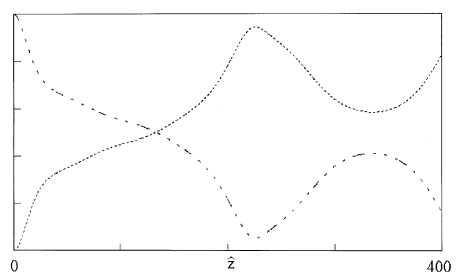

(i)

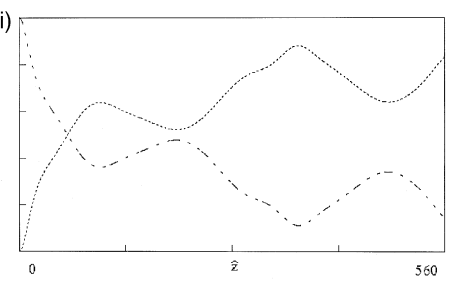

(I)

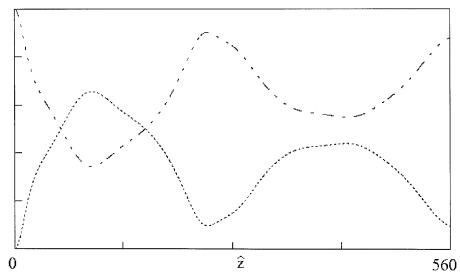

Fig. 1. Evolution of the values of the relative energies $N_{1,2} / N$ in each channel as a function of $\hat{z}$ for the initial profile (4) with $N=2$; dashed: channel 1, dotted: channel 2. The normalized coupling factor $\hat{\kappa}$ increases for (a)-(1). Observe in particular the two sharp switching transitions in (d), (e) and (j), (k). 
is seen to shift to the right for increasing $\hat{\kappa}$, i.e. $\hat{z}_{\max }$ increases.

$(e),(f)$ : switching. In these two graphs switching occurs. Similarly to the above, now a distance $\hat{z}_{\max }$ of maximum energy transfer between the two channels can be defined. At the maximum the relative energy in the second channel $N_{2} / N$ equals approximately 0.9 . An increase in the coupling parameter increasing $\hat{\kappa}$ now leads to a shift of the top to the left, i.e. to a decrease of $\hat{z}_{\max }$.

$(g),(h),(i),(j)$ : switching. Switching is still observed, with some differences from (e), (f) above, however. First the direction of the shift to the left of the maximum of the $\mathrm{N}_{2}$-curve, as observed in Fig. 1(e)-1(f), reverses. Thus $\hat{z}_{\max }$ attains a minimum, viz. $\hat{z} \approx 240$. Furthermore, in the process a second, lower maximum splits off. This lower maximum disappears (cf. (h)-(k)), without having obtained a relevant role. Second a new maximum develops at $\hat{z} \approx 100$. However, the corresponding energy reaches a maximum of only $N_{2} / N \approx 0.6$. Anticipating on the design of a routing device, for which a large energy transfer is required, this maximum is irrelevant for this purpose.

$(k),(l)$ : non-switching. Further increasing $\hat{\kappa}$, one finds the graphs (k), (1). They should be classified as nonswitching: although switching of the original pulse occurs at a distance of approximately $\hat{z} \approx 100$ in these figures, the energy transfer between the two cores at $\hat{z} \approx 100$ is relatively small viz. equals approximately 0.6 ; at $\hat{z} \approx 480$ and $\hat{z}=200$, respectively, most energy is back in the original channel. The relevant maximum of $N_{1} / N$, at which the energy exchange between the two cores is minimum, viz. 0.1 , shifts to the left, i.e. $\hat{z}_{\max }$ decreases for increasing $\hat{\kappa}$.

Summarizing, in the graphs in Fig. 1 two extremely sharp switching transitions are observed. The first one occurs for $\hat{\kappa}$ in the range $0.136591<4 \hat{\kappa}<0.136592$, graphs (d), (e), and the second one in $0.142052<4 \hat{\kappa}<$ 0.142053 , graphs $(\mathrm{j}),(\mathrm{k})$. Note in particular that for increasing $\hat{\kappa}$, i.e. for decreasing $N$, the first transition is from the non-switching to the switching situation, while the second concerns the transition from switching to nonswitching. Thus the $\hat{\kappa}$-region under consideration is divided in three parts corresponding to non-switching, switching and non-switching respectively.

These conclusions are concisely represented in Fig. 2, showing $\hat{z}_{\max }$ and the corresponding energies in the channel with the highest energy as a function of the normalized coupling factor $\hat{\kappa}$. Some care is necessary to define $\hat{z}_{\max }$. In the first six graphs of Fig. $1 \hat{z}_{\max }$ there is no problem. In the graphs $(\mathrm{g})-(\mathrm{j})$ however, two or more maxima are observed. The maximum at $\hat{z} \approx 100$ is ignored, since its energy is too low to be relevant for switching, as was stated already. The maximum at $\hat{z} \approx 240$ evolves into a double maximum, of which we take the highest to determine $\hat{z}_{\max }$. Note that this may lead to a small discontinuity in the graph $\hat{z}_{\max }$ as a function of $\hat{\kappa}$, when one maximum takes over the role of the other (here this does not happen, however).

The main feature of the $\hat{z}_{\max }-\hat{\kappa}$ curve is the presence of two sharp peaks. They divide the $\hat{\kappa}$-region in the

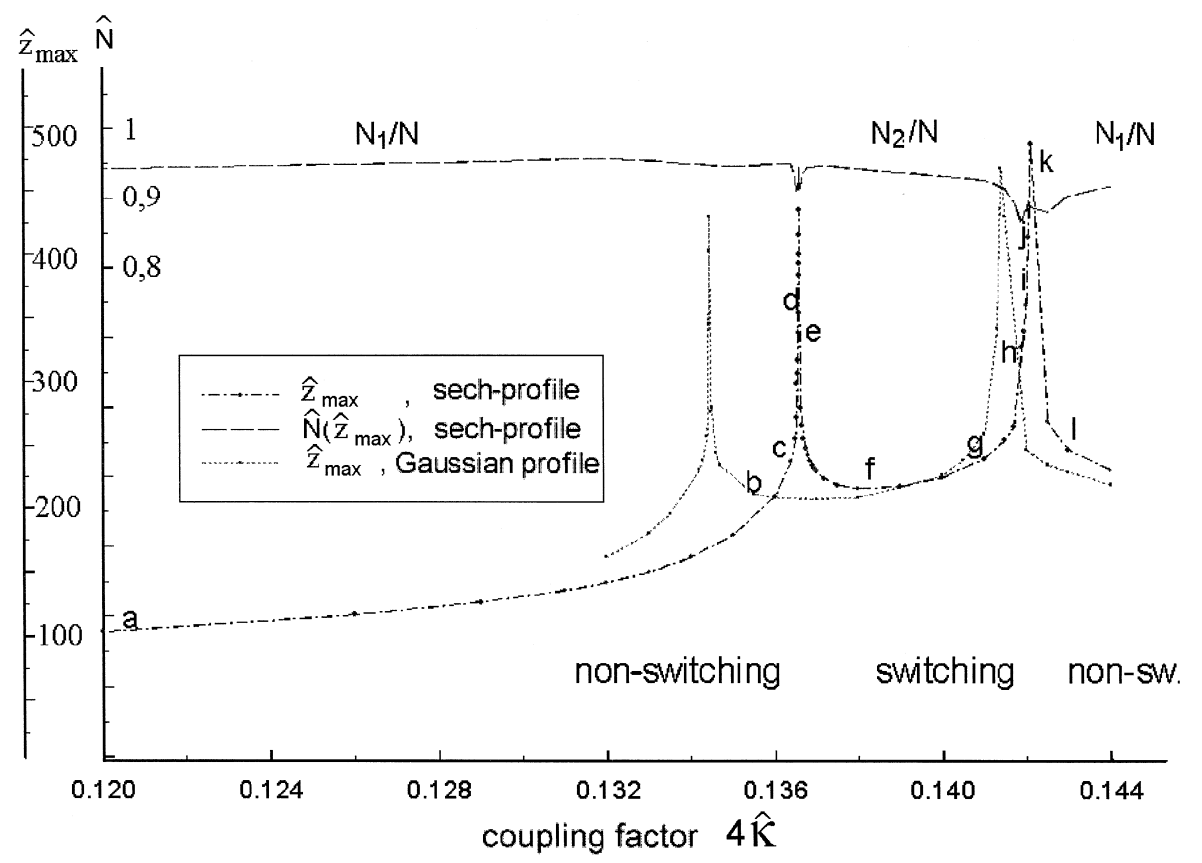

Fig. 2. Dotted curve: the distance $\hat{z}_{\max }$ of minimum or maximum energy transfer (non-switching or switching respectively) as function of $\hat{\kappa}$. The thick dots represent specific calculations. Dashed: the corresponding relative energies $N_{1} / N$ and $N_{2} / N$. Note in particular the sharp peaks and the high value of the relative energies. 
switching and non-switching subregions, as mentioned above. Their sharpness demonstrates the sharpness of the switching process.

In this figure also the relative energy $N_{1} / N$ and $N_{2} / N$ at the distance $\hat{z}_{\max }$ is shown for non-switching and switching respectively. Clearly these values do not become less than about 0.9 in the $\hat{\kappa}$-region under consideration, which is large with respect to the width of the $\hat{z}_{\max }$-peaks.

In order to test the robustness of these results for a small change of the initial condition, calculations were performed starting from the Gaussian field

$$
\begin{gathered}
u_{1}=a \exp \left(-b^{2} t^{2}\right), \quad u_{2}=0, \\
\text { with } a=0.474, b=0.282 .
\end{gathered}
$$

The parameters $a$ and $b$ are chosen so that for given $N=1$ the Hamiltonian of (1) is maximal (cf. Ref. [1]). This makes (5) close to expression (4), but not exactly equal to it of course. The resulting $\hat{z}_{\max }$-curve near the first peak is shown in Fig. 2. Clearly the effect is only a small shift of the position of this peak, i.e. $\leq 2 \%$ in $\hat{\kappa}$. The corresponding values of $N_{1,2}$ are not displayed, since they coincide almost with the values obtained for (4). Finally it must be remarked that the precise position of the peaks are somewhat influenced by the choice of the step width in the integration procedure. Qualitative features of the graph remain the same, however.

\section{A sharp switching characteristic}

Making use of the extremely narrow transition region between switching and non-switching as shown in Fig. 2, one should be able to improve the sharpness of (half) beat length switching devices (cf. Refs. [2,3]) at the cost of a much longer device, however. Both sharp transitions in Fig. 2 can in principle be applied to design such a coupler. As is apparent from this graph, sharp switching is obtained using a coupler length above $\hat{z}=240$, say. The sharpness is determined by the width of the peak at the $\hat{z}$-level.

This is demonstrated in Fig. 3, which shows the relative energy $N_{1} / N$ of the pulse in the original channel as a function of $\hat{\kappa}$, at two fixed distances, $\hat{z}=240$ and $\hat{z}=280$. The relative energy $N_{1} / N$ in these curves varies from about 0.9 to 0.1 within a very narrow $\hat{\kappa}$-range: the relative width $\Delta \hat{\kappa} / \hat{\kappa}$ is less than $0.2 \%$, which implies a relative difference in the switching control parameter of $\Delta N / N<$ $0.1 \%$. The sharp variation of $N$ is particularly observed at the first transition, which is illustrated by the enlargement of the rectangle.

\section{Field profiles at $\hat{\mathbf{z}}=\mathbf{2 4 0}$ and their evolution}

Anticipating on the application of this process in a routing device, we are interested in the shape of the signal
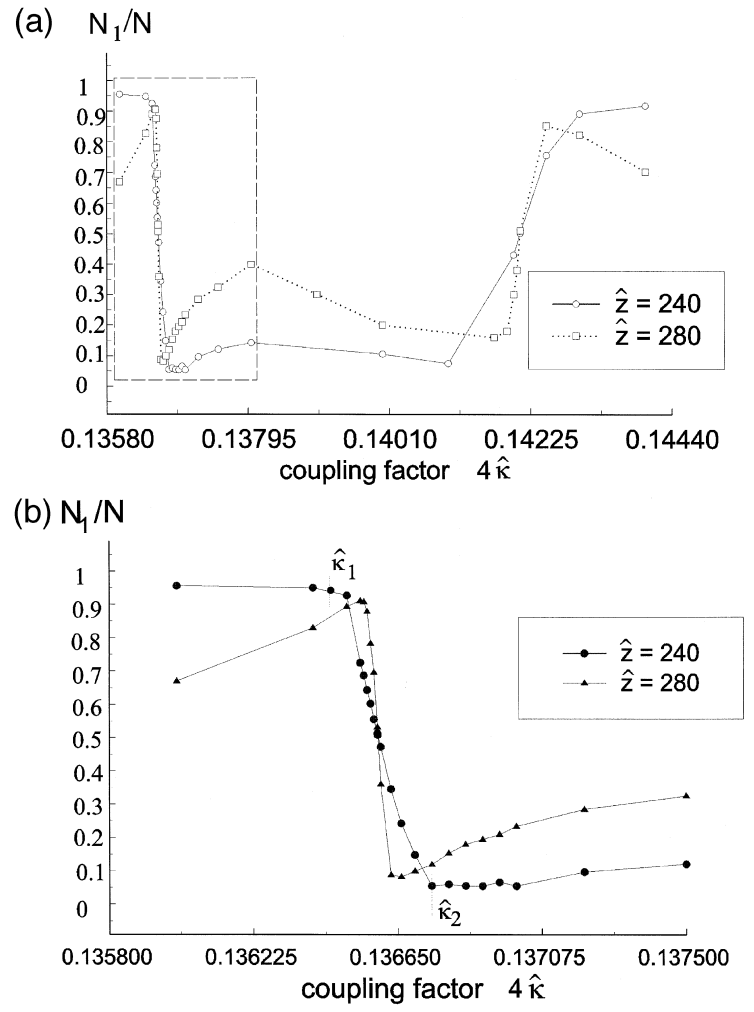

Fig. 3. Relative energy $N_{1} / N$ at two different positions $\hat{z}=240$ and $\hat{z}=280$ as a function of $\hat{\kappa}$. The initial condition at $\hat{z}=0$ is a soliton in channel 1 . In (b) an enlargement is shown of the rectangle in (a).

at its end and the further evolution of the signal along two uncoupled channels.

In Fig. $4 \mathrm{a} 1,4 \mathrm{a} 2$ the field profiles at a distance $\hat{z}=240$, the length of a possible coupler, are shown. Fields at the left are non-switching, fields at the right are switching. These fields will evolve from here with the two channels uncoupled $(\hat{\kappa}=0)$, i.e. each in a single NLS fiber. The result of the evolution at $\hat{z}=1040$ is shown in Fig. 4b1, $4 \mathrm{~b} 2$. The primary observation is that in either case the main pulse remains a pulse of almost the same height and width. The small pulses, however, seem to disperse.

More details of the evolution process are shown in Fig. $4 c 1,4 c 2$. In either case the height of the main pulse oscillates and its shape converges to a stable pulse. This is the typical behavior of a near first order soliton initial condition evolving according to NLS. The small pulse appears to fall apart into radiation: seemingly the initial condition does not contain a soliton in the 'inverse scattering' sense [13].

In conclusion, the switching process of a soliton initial condition, yields a soliton again, with $\approx 90 \%$ of the energy of the original soliton. Another remarkable fact is that the corresponding graphs $4 \mathrm{a} 1,4 \mathrm{a} 2 ; 4 \mathrm{~b} 1,4 \mathrm{~b} 2 ; 4 \mathrm{c} 1,4 \mathrm{c} 2$ are almost the mirror image of each other. Thus an ex- 
(a)
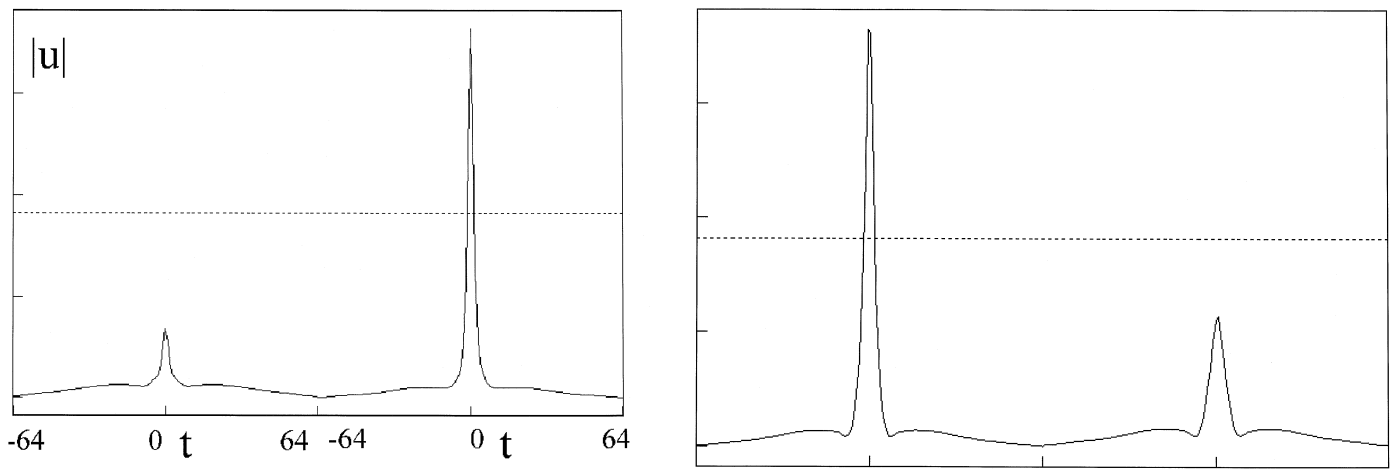

(b)
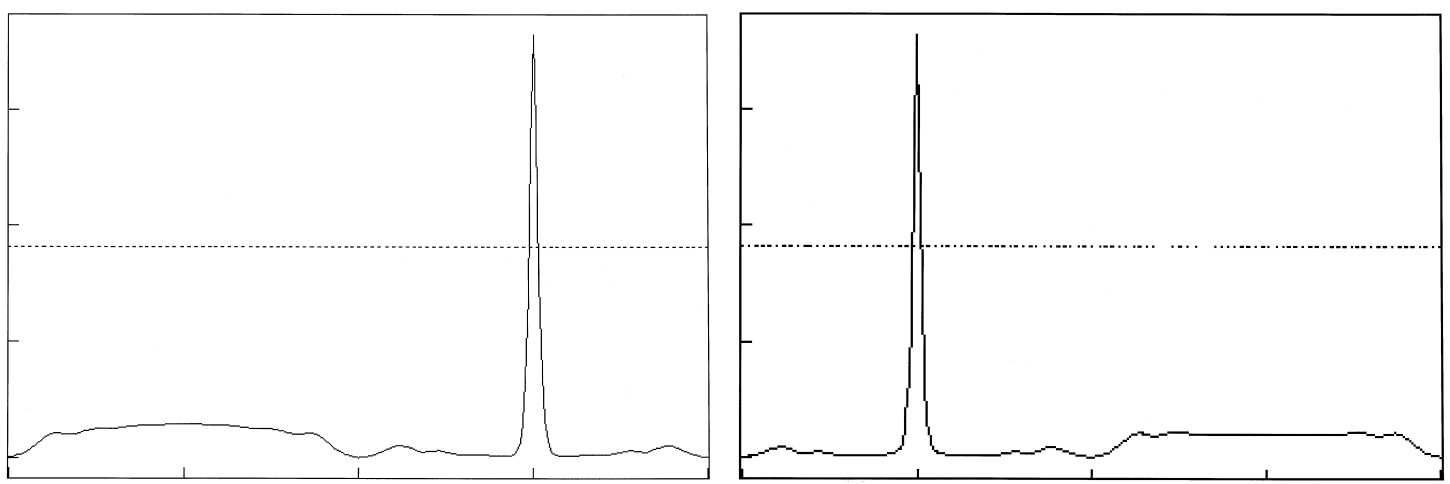

(c)
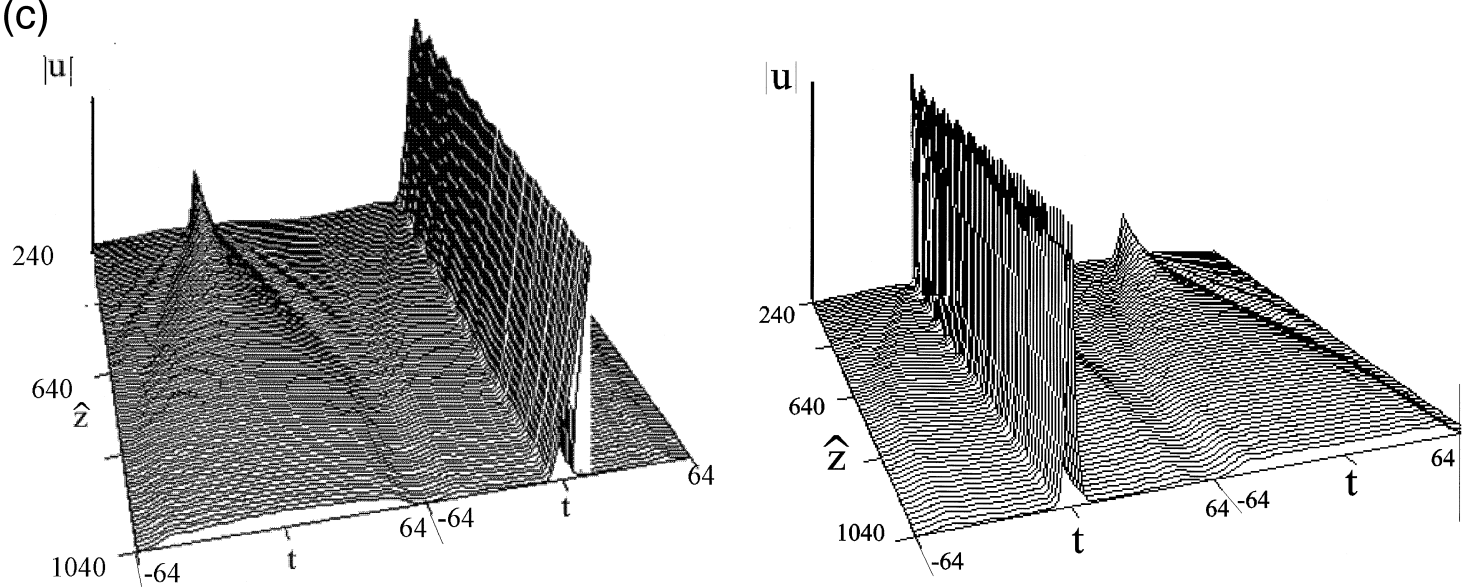

Fig. 4. Field amplitudes at $\hat{z}=240$ (a), $\hat{z}=1040$ (b) and in between (c) for a non-switching and a switching value of $\hat{\kappa}, \hat{\kappa}=\hat{\kappa}_{1}=0.03411$ and $\hat{\kappa}=\hat{\kappa}_{2}=0.03418$, marked in Fig. 3(b).

tremely small variation in this initial energy can result in a reversed signal at the end of the fiber.

\section{Conclusions and discussion}

The switching of optical solitons in a nonlinear fiber coupler was investigated by calculating numerically the evolution of an input signal, consisting of a soliton in one of the cores, and no signal in the other. The switching control is the signal energy $N$.

It was found that efficient and extremely sharp switching is possible: switching takes place within a relative energy range of $0.1 \%$. For a non-switching signal $90 \%$ of the energy returns to the original channel, and for a 
switching signal $90 \%$ is transferred. The main transferred signal appears to be close to a soliton, whereas the field in the other channel does not contain a soliton, and disperses when it propagates in a single fiber. This transition takes place at coupler length and coupling constant given by

$\hat{z}=z N^{2} \geq \hat{z}_{\mathrm{sW}} \equiv 240, \quad \hat{\kappa}=\kappa / N^{2}=\hat{\kappa}_{\mathrm{sw}} \equiv 0.03415$.

These data yield immediately the coupling factor and a lower bound for the length for given $N$. With a Gaussian near soliton initial profile the results are essentially the same. Only the transition point shifts by about $1 \%$ to a lower value $\kappa / N^{2}=0.03360$.

The transition switching/non-switching happens for decreasing $N$. Remarkably enough, a second transition was observed for increasing $N$. At the same coupler length the sharpness is then about $0.5 \%$ and the transition occurs for $\kappa / N^{2}=0.03551$.

A feature crucial to explain the phenomena described here is found in the instability of the symmetric soliton in the coupled fiber for the relevant values of $\hat{\kappa}$. For further details the reader is referred to Ref. [1].

These observations can be possibly used to develop a sharply switching routing device or a sensor, much sharper than the usual (half) beat length couplers, but also much longer. To find the actual properties observe that the transformations that relate (1) with the corresponding equations in physical variables (see e.g. Ref. [3], and Refs. $[12,13]$ for the single NLS) are given by

$z=Q Z, \quad t=\sqrt{Q}\left(T-k_{0}^{\prime} Z\right) / \sqrt{-k_{0}^{\prime \prime}}, \quad \kappa=K / Q$.

Here capitals denote the physical variables and $Q=k_{0} n_{2} \alpha$, with the geometrical reduction factor $\alpha=1 / 2$. Furthermore, if the width $w$ of the soliton pulse in (4) is defined by $w N / 2=1.76$, the width $W$ of the physical pulse is given by $W=3.52 \sqrt{-k_{0}^{\prime \prime} / Q} / N$. With these relations one eliminates $N$ from (6) and, with (7), one finds for the length and coupling constant for switching of a pulse of duration $W$

$Z \geq-k_{0}^{\prime \prime-1} \frac{W^{2}}{12.4} \hat{z}_{\mathrm{sw}}, \quad K=-k_{0}^{\prime \prime} \frac{12.4}{W^{2}} \hat{\kappa}_{\mathrm{sw}}$.

For a twin-core fiber coupler with zero dispersion wavelength $1.3 \mu \mathrm{m}$ and with carrier wavelength of $1.5 \mu \mathrm{m}$ one finds $k_{0}^{\prime \prime}=-1.9 \times 10^{-26} \mathrm{~s}^{2} \mathrm{~m}^{-1}$. This yields for a pulse of $1 \mathrm{ps} Z \geq 1000 \mathrm{~m}$ and $K=8 \times 10^{-3} \mathrm{~m}^{-1}$. Similarly for a pulse of $100 \mathrm{fs}$ one finds $Z \geq 10 \mathrm{~m}$ and $K=8 \times 10^{-1}$ $\mathrm{m}^{-1}$ respectively. The large variation in these values is due of course to the quadratic dependance of these quantities on the pulse width as expressed in (8). One may compare these lengths with the widely investigated linear half beat length couplers of length $\pi / 2 K$. For the $K$ values given above one finds $200 \mathrm{~m}$ and $2 \mathrm{~m}$ respectively. Note that also the value of $k_{0}^{\prime \prime}$ depends strongly on the wavelength of the carrier wave, which might be of practical value to adapt the required length of the coupler.

In particular for a pulse width larger than $1 \mathrm{ps}$, when the device should be fairly long, one may wonder to what extent the results presented here are influenced by the fact that the CNLS equations (1) do not give a perfect description if the real coupler. Our guess is that this primarily will influence the given numbers somewhat, and that the phenomenon qualitatively will not change. The main reason is that a soliton travelling in a single fiber is proven to be extremely robust to perturbations, including the conservation of a uniform phase. This makes it suitable for switching purposes, cf. Ref. [12]. For a switching soliton this robustness is not investigated extensively, and it seems worthwhile to do so.

\section{References}

[1] T.P. Valkering, P.T. de Boer, H.J.W.M. Hoekstra, Soliton dynamics in directional couplers, Physica D 123 (1998) 223.

[2] G.I. Stegeman, E.M. Wright, Opt. Quantum Electron. 22 (1990) 95.

[3] M. Romagnoli, S. Trillo, S. Wabnitz, Opt. Quantum Electron. 24 (1992) 1237.

[4] S.R. Friberg, Y. Silberberg, M.K. Andrejco, M.A. Saifi, P.W. Smith, Appl. Phys. Lett. 51 (1987) 1135.

[5] J. Wilson, G.I. Stegeman, Opt. Quantum Electron. 24 (1992) 1325.

[6] C. Paré, M. Florjanczyk, Phys. Rev. A 41 (1990) 6287.

[7] I.M. Uzunov, R. Muschall, M. Gölles, Y.S. Kivshar, B.A. Malomed, F. Lederer, Phys. Rev. E 51 (1995) 2527.

[8] N. Akhmediev, A. Ankiewicz, Solitons, Nonlinear pulses and beams, Chapman \& Hall, London, 1997.

[9] N. Akhmediev, A. Ankiewicz, Phys. Rev. A 47 (1993) 3213.

[10] J.M. Soto-Crespo, N. Akhmediev, Phys. Rev. E 48 (1993) 4710.

[11] E.M. Wright, G.I. Stegeman, S. Wabnitz, Phys. Rev. A 40 (1989) 4455.

[12] G.P. Agrawal, Nonlinear Fiber Optics, Academic Press, San Diego, 1995.

[13] A. Hasegawa, Y. Kodama, Clarendon Press, Oxford, 1995. 